\title{
Frequency and time profiles of metric wave isolated Type I solar noise storm bursts at high spectral and temporal resolution
}

\author{
G. A. Shanmugha Sundaram ${ }^{1,2 \star}$ and K. R. Subramanian ${ }^{2}$ \\ ${ }^{1}$ Joint Astronomy Programme, Department of Physics, Indian Institute of Science, Bangalore - 560012, KA, India \\ ${ }^{2}$ Indian Institute of Astrophysics, Koramangala, Bangalore - 560034, KA, India
}

Accepted 2005 February 14. Received 2005 February 2; in original form 2004 May 18

\begin{abstract}
Type I noise storms constitute a sizeable fraction of the active Solar radio emission component. Observations of isolated instances of such bursts, in the swept-frequency mode at metric wavelengths, have remained sparse, with several unfilled regions in the frequency coverage. Dynamic spectra of the burst radiation in the $30-130 \mathrm{MHz}$ band, obtained from the recently commissioned digital High Resolution Spectrograph at the Gauribidanur Radio Observatory, have unravelled in explicit detail the temporal and spectral profiles of isolated bursts thanks to the instrument's superior frequency and time resolution. Apart from presenting details of their fundamental emission features, the time- and frequency-profile symmetry, with reference to custom-specific Gaussian distributions, has been chosen as the nodal criterion to statistically explain the state of the source regions in the vicinity of magnetic reconnections, the latent excitation agent that contributes to plasma-wave energetics, and the quenching phenomenon that causes damping of the burst emission.
\end{abstract}

Key words: plasmas - radiation mechanisms: non-thermal - instrumentation: spectrographs - methods: data analysis - Sun: radio radiation.

\section{INTRODUCTION}

Type I radio noise storms head the list of solar events discovered at metric wavelengths (Krucker et al. 1995). Noise storms rage over a prolonged duration, and appear as intense, narrow-band bursts, superposed on a low-intensity broadband continuum, in the $30-400 \mathrm{MHz}$ range. Either component of the noise storm radiation has a very high degree of ordinary mode circular polarization $(\sim 100$ per cent), and is widely believed to be generated by the plasma emission mechanism. Ever since their discovery (Hey 1946), they have been among extensively studied solar radio features. Over the years, several notable theoretical and observational works on this particular phenomena have been performed (see Wild \& McCready 1950; Kundu 1965; Kerdraon 1973; Mangeney \& Veltri 1976; Melrose \& Stenhouse 1977; Elgarøy 1977; Krüger 1979; Melrose 1980a,b; Benz \& Wentzel 1981; Kai, Melrose \& Suzuki 1985).

Features such as short duration, high-polarization, narrow bandwidth, a random distribution in frequency and height associated with Type I noise storm bursts, have been considered as evidence for the radiation process to be of plasma origin, occurring in the vicinity of the local plasma frequency or at its harmonic(s). The bursts are caused owing to excitation of the upper hybrid waves by a trapped population of energetic non-thermal electrons, bounded by the mag-

^E-mail: sga@physics.iisc.ernet.in netic field lines of the coronal plasma (Bárta \& Karlický 2001). The electrons that are in the vicinity get accelerated along the magnetic field, and such an anisotropic beam tends to become unstable. The bursts occur when non-thermal electron densities approach a threshold value, and the phenomenon exhibits significant sporadicity. The threshold density values show a downward trend with frequency, and fluctuations in the densities of either the superthermal electron beam or the ambient coronal plasma, determine the bandwidth and duration of Type I bursts.

We present observational results on this rapidly changing time and frequency component of the metric Type I noise storm radiation, along the lines of their dynamic spectral characteristics and the implied plasma emission mechanism. The section that follows is an account of the High Resolution Spectrograph (HRS), and the flux calibration scheme adopted for the observed dynamic spectrum of the bursts. Unique traits associated with the isolated bursts, such as the bandwidth of emission, duration and flux distribution, are interpreted in terms of the coronal plasma environment in the associated source region. A statistical study is performed on the distribution of frequency and time profile shapes, with reference to customized Gaussian profiles, in order to explain the excitation and damping mechanisms of plasma waves, and the agent responsible for shaping the observed profiles. The last section explains the spectrum and profile shapes, from discussions based on the strikingly corroboratory nature of the isolated metric bursts with spikes observed at relatively higher frequencies, with generic attributes to 
elementary radiation processes in the coronal source regions of this plasma emission phenomenon.

\section{INSTRUMENT DETAILS AND OBSERVATIONS}

\subsection{High-resolution spectrograph and antenna system}

The HRS includes one group of antennas of the Gauribidanur Radioheliograph (GRH Ramesh et al. 1998) and a spectrum analyser. An array of four log-periodic dipole antennas (LPDAs) with their E-planes aligned mutually (along the E-W direction) and stacked along the $\mathrm{H}$ planes (along the $\mathrm{N}-\mathrm{S}$ direction), constitutes the basic element for signal interception. The $(\mathrm{E}-\mathrm{W})$ beam is $90^{\circ}$, and this offers about $6 \mathrm{~h}$ of continuous spectral observations of the Sun in the frequency band of $30-150 \mathrm{MHz}$. The beamwidth along the (N-S) direction varies from $6^{\circ}$ at $150 \mathrm{MHz}$ to $30^{\circ}$ at $30 \mathrm{MHz}$; hence the Sun remains practically unresolved over the entire operating frequency range of the spectrograph.

The amplified radio signals are fed to a computer-controlled, commercial off-the-shelf spectrum analyser, that accomplishes the role of multifrequency solar radio spectrum analysis. The device has a logarithmic response, with a frequency sampling rate of about $41 \mathrm{~ms}$. The instantaneous bandwidth (or frequency resolution of the spectrograph) for this particular investigation on Type I bursts works out to $\approx 250 \mathrm{kHz}$. A GPIB/RS-232 at the communication port enables the acquisition of data by a desktop computer in a three-way frequency versus time versus signal amplitude (arbitrary units) format termed the dynamic spectrum, for offline spectrum analysis.

\subsection{Dynamic spectral data and flux calibration}

The output of the HRS is of a data type, that has the detected signal in arbitrary units of signal power, acquired in the frequency range $30-130 \mathrm{MHz}$, across the 401 equally spaced frequency channels, for a duration of $6 \mathrm{~h}$. This transit-mode observation schedule for the Sun has been in vogue since 2002 June, with each day's run commencing at 03:30 UT and ending at 09:30 UT. Dynamic spectra of the Sun were obtained on days 2002 July $15,16,17,18,28$ and 30 and 2002 August 13, 17 and 18, because Type I burst activity was found to be very intense on those days.

The galactic background has been used in gain calibration of the spectral channels. Appendix A describes the procedure adopted for calibration in greater detail.

A suitable algorithm was designed to 'harvest' isolated cases of Type I noise storms bursts, that appear as intensity enhanced bursts in the frequency-time plane, the criterion for classifying the desirable spectral event being its enhanced intensity level as against the background, its emission duration and bandwidth. The broadband continuum component of the noise storm spectrum was essentially subtracted as background radiation from the dynamic spectral records, as were the long time-scale modulations; this enhanced the dynamic range for detection of the burst component in noise storms.

\section{CHARACTERISTICS OF THE ISOLATED NOISE STORM BURSTS}

In this section, the characteristics of isolated instances of Type I bursts, of the kind shown in Fig. 1, such as their distribution with central frequency, bandwidth, duration (lifetime) and calibrated flux, observed in the dynamic spectral records for the few select days
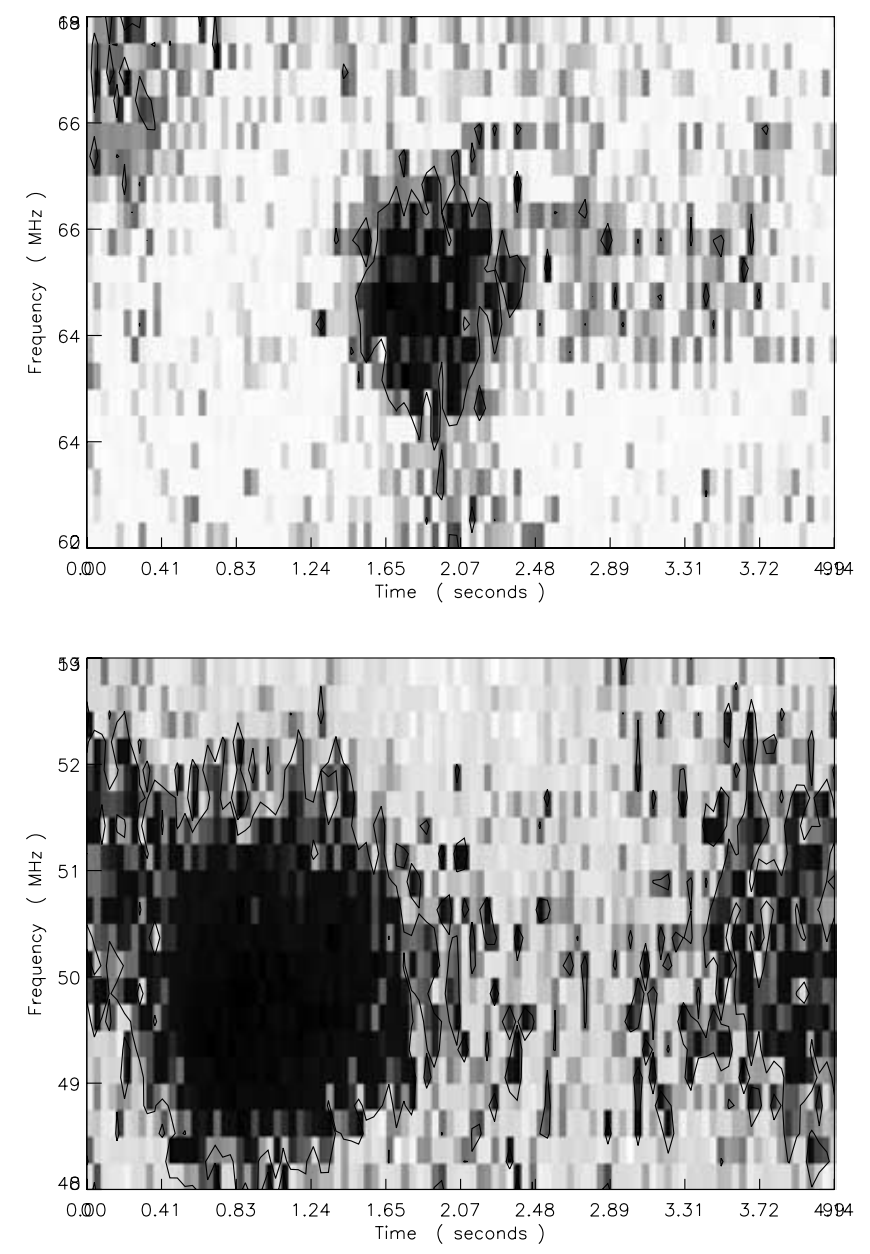

Figure 1. Dynamic spectral records of isolated Type I bursts that occurred on 2002 July 28.

of 2002 July and August, are reported. A collection of 39 individual instances of isolated bursts was chosen for the study; they were well resolved in time and frequency, and the compilation includes only bursts with profiles that occurred unambiguously in the expected typical bandwidth range. The peaks were determined by looking for strong enough local maxima above the noise level $(\geqslant 5 \sigma)$, and a constant background (representing the quiet Sun and other slow-varying baseline components) was subtracted. In the broad frequency range spanning $100 \mathrm{MHz}$, the study of frequency and time profiles of these rapidly evolving structures, recorded at a high digitization rate, would be fundamental to the analysis of profile shapes in terms of the rise and decay phases, and hence provide details on the source region for plasma emission.

\subsection{Distribution of radio flux of isolated Type I bursts}

The distribution of isolated Type I noise storm bursts with their calibrated radio flux, reveals a peak in the distribution at $\sim 25$ solar flux units (sfu). The result concurs with a similar peak flux occurrence amid $20-40$ sfu in the $70-130 \mathrm{MHz}$ band (Wild 1950). There occurs an inherent uncertainty, associated with the dynamic range of the spectrograph, in the detection and estimation of the radio flux of low-intensity bursts, during periods of intense noise storm activity; this would leave the flux-poor bursts as being unaccounted for, during such instances. Yet, it has little impact on the detection of 
flux-rich bursts, thereby implying that a significant number of bursts during the period of noise storm activity under consideration, are of the low-intensity type.

The narrowband bursts exhibit frequency-independent energy characteristics at metric wavelengths; about 75 per cent of the observed bursts occur in the $42-85 \mathrm{MHz}$ range. This trend can be interpreted in terms of the high degree of constancy in the density and temperature parameters, along the path of the exciting disturbance in the active source region, and in a direction parallel to that of the solar surface (as with the case of bursts generated near the apex region of evolving magnetic loops).

\subsection{Frequency-profile analysis: distribution of isolated bursts with central frequency and bandwidth}

A distribution was noticed in the isolated bursts as a function of their bandwidth and fractional bandwidth (FBW). Of the 39 cases, more that half (i.e. 54 per cent) the bursts have a bandwidth that is below $3 \mathrm{MHz}$; 69 per cent below $3.5 \mathrm{MHz}$ and 82 per cent below $4 \mathrm{MHz}$. These values compare favourably with $2.8 \mathrm{MHz}$, observed for the half-power bandwidth in the 70-130 MHz range (Wild 1950; Elgarøy 1977 and references therein), for the case where a Gaussian distribution is the most likely frequency-profile fitting. This indicates that the majority of the isolated Type I bursts in this sample, were emitted at half-power bandwidths less than $4 \mathrm{MHz}$, with the peak in the distribution being in the $2-2.5 \mathrm{MHz}$ restricted band. Another notable feature was the near absence of fundamentalharmonic pairs among the burst events chosen for this study. As the bandwidth per channel $(\approx 0.25 \mathrm{MHz})$ is considerably lower than the observed bandwidths, significant errors in this aspect can be discounted.

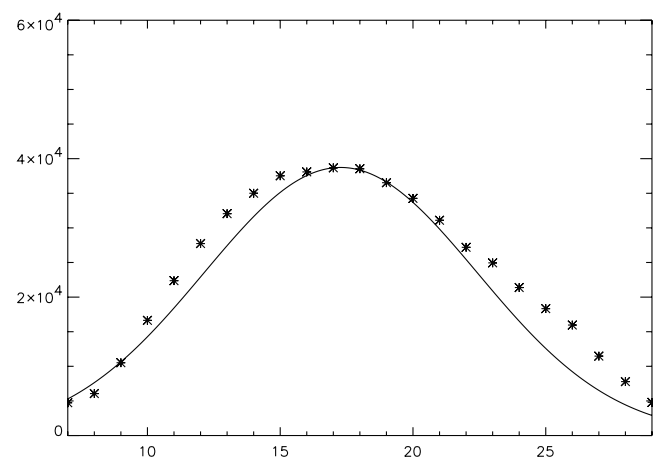

[a]

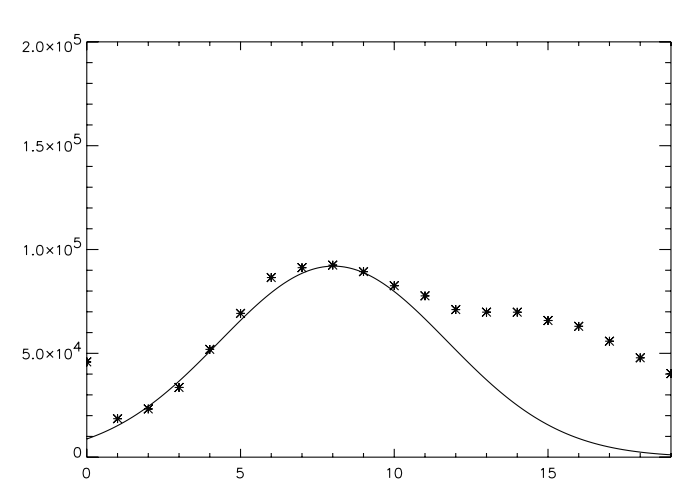

[c]

[b]

$\Delta v_{\mathrm{fr}}=2\left(\frac{f_{\mathrm{H}}-f_{\mathrm{L}}}{f_{\mathrm{H}}+f_{\mathrm{L}}}\right) \equiv \frac{\Delta f}{f_{\mathrm{c}}}$,

where $f_{\mathrm{H}}, f_{\mathrm{L}}$ and $f_{\mathrm{c}}$ are respectively the high, low and central frequencies (in $\mathrm{MHz}$ ) for each burst event, and $\Delta_{\mathrm{f}}$ is its bandwidth. The peak, in the distribution of burst events with FBW, for $\sim 50$ per cent of samples considered, occurs at an instantaneous FBW of 4 per cent, and this meets the criterion, for the Type I bursts considered, of narrow-band emission characteristics.

The analysis of dynamic spectral records on noise storm bursts indicates an extremely low degree of frequency drift, and the absence of harmonics. A quantitative study was performed, in order to estimate the degree of deviation from symmetry, in cases where Gaussian fittings were performed to the time-averaged frequency profiles. A typical burst profile in frequency has a shape that favours the choice of a Gaussian fitting, because of the following points. (i) The spectrum of a burst is symmetric in most cases, at least within the frequency domain of the half-maximum points (though deviations from the Gaussian curve are noticeable, especially at the wings). (ii) The spectral behaviour of the flux, either side of the peak frequency, appears bell shaped. The Gaussian functions are of the standard form

$g(x)=\frac{1}{\sigma \sqrt{2 \pi}} \exp \left[-\frac{1}{2}\left(\frac{x}{2 \sigma}\right)^{2}\right]$,

where $\sigma \sqrt{2 \pi}$ is the weighting factor in the distribution, and the standard deviation $\sigma$ influences the Gaussian width, which in this case would be the half-power bandwidth of the burst event, ' $x$ ' being the length-scale of the distribution.
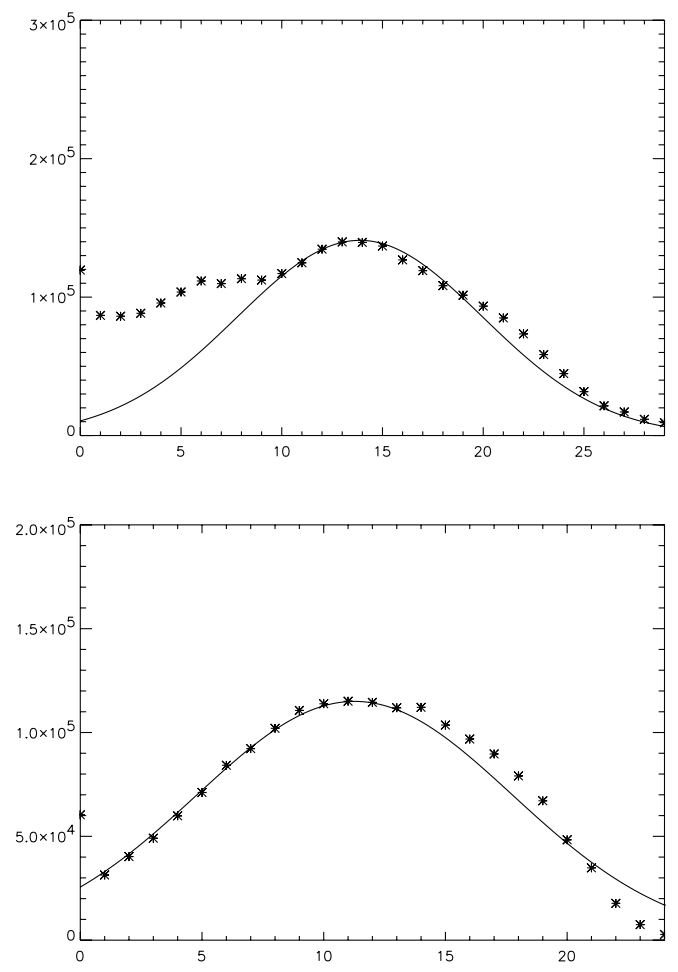

[d]

Figure 2. The three kinds of isolated Type I noise storm bursts, according to their time-averaged frequency profiles. The abscissa is in terms of frequency channels, with adjacent channels being $250 \mathrm{kHz}$ apart; the ordinate axis is in uncalibrated flux units. Subplots (a) and (d) indicate symmetry, while (b) and (c) show trailing and leading edge asymmetry, to the fitted Gaussian distribution. 
Fig. 2 depicts the three kinds of profiles encountered in the study viz., the symmetric (subplots a and d), the ascending-limb asymmetry (subplots b) and the descending-limb asymmetry (subplots c). Asymmetry in the frequency profiles is found to be manifested as an enhanced tail portion straggling the leading or trailing limb of the Gaussian profile fitted to the time-averaged data points. Among the Type I bursts detected, which conform to one of the possible three distribution patterns, the majority ( $\approx 54$ per cent) of events follow a symmetric, Gaussian frequency distribution, while $(\approx 26$ per cent) belong to the ascending-limb asymmetry and $(\approx 21$ per cent) to the descending-limb asymmetry. The insensitivity of the bandwidth regarding frequency, and the occasional observation of extremely small bandwidths, are reflected in the near constant scatter of the FBW with the central frequency of the bursts. This strongly indicates that the emission process is of the narrowband type, and that the observed bandwidth of a burst is its natural width owing to the emission process and not a consequence of inhomogeneities in the source region.

\subsection{Time-profile analysis: distribution of lifetimes of isolated bursts}

The duration of a burst may be given by the time interval between its very beginning (which is usually fairly well defined) and its fall below the $3 \sigma$ limit of the instrument, above the background level. This would describe the total duration, homogeneously restricted to a given sensitivity. Among the bursts chosen with definite start and end times, the differing peak flux levels, as well as subjective estimates of the background, cause a considerable scatter of the result, which is referred to as the 'total duration' of isolated bursts. The duration measures widely used in the literature are: the time interval between the half-peak flux levels (half-power duration), the time interval between the 1/e-peak flux levels (natural duration) and the time interval between 1/10-peak-flux levels (Güdel \& Benz 1990 , and references therein).

The natural duration is on an average close to the physically meaningful duration of the exciting agent arising as a consequence of the plasma instability in the source region for the bursts. The peak in the distribution of isolated Type I bursts with their lifetime occurs at $1.5 \mathrm{~s}$, and this agrees with values of $1.2 \mathrm{~s}$ (Warwick \& Dulk 1969), $1.5 \mathrm{~s}$ (Wild 1950 in the 70-130 MHz band) and 1.01 s (Sastry 1969), for the lifetime of bursts of Type I noise storm radiation.

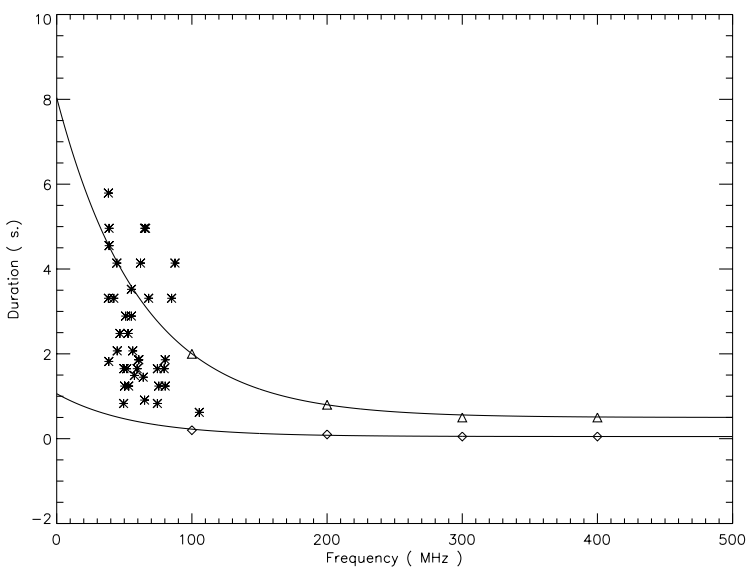

Figure 3. Duration of isolated Type I noise storm bursts as a function of frequency. The upper and lower curves are exponential-decay fittings to observations as in (Elgarøy \& Eckhoff 1966). Asterixes stand for the HRS observations.
The variation in mean duration of Type I bursts with their observation frequency is depicted in Fig. 3. The upper and lower exponential decay curves are fittings made to the observations of noise storm bursts in the $100-500 \mathrm{MHz}$ frequency range (Elgarøy \& Eckhoff 1966; Mangeney \& Veltri 1976, and references therein); they indicate the upper and lower limit on the duration of the bursts. The Type I burst events observed with the HRS are shown by asterixes. It is notable that about 83 per cent of the burst events occur within and about the confines of the two bounding curves, which have the form

$t_{\mathrm{u}}=(0.5+\exp [2.02-(f / 62)]) \quad \mathrm{s} \quad$ and

$t_{1}=(0.05+\exp [0.01-(f / 57)]) \quad \mathrm{s}$.

Here, $f$ is the frequency in $\mathrm{MHz}$, and $t_{\mathrm{u}}$ and $t_{1}$ are the durations as defined by the upper and lower curves of Fig. 3, over the frequency range.

Based on their time profile, noise storm bursts are defined as follows.

(i) Structures with time constants of a few hundred milliseconds.

(ii) Identifiably short-lived, narrow-band single bursts.

(iii) Typical duration, bandwidth/duration ratio and distribution in the time-frequency plane.

(iv) Near irregular distribution in the frequency-time plane, showing no strict time or frequency correlation among the different bursts.

The typical time profile for bursts shows a steep rise, a short saturation phase, followed by exponential decay. The essential criteria are a smooth increase and decrease in the profile. Considering the decay phase of individual bursts, an exponential decay law suggests a physical description in terms of a suitable damping mechanism, either inherent to the ambient plasma or to the exciter mechanism itself that accounts for it. If it can be shown that the e-fold decay times are somehow related to the burst duration or to the peak flux values on a fixed frequency, relating the damping mechanism to the exciter mechanism could be envisaged. If, on the other hand, the decay rates do not correlate with the burst durations, but tend to a constant value, a damping mechanism independent of the radiation process ought to be assumed. The time profiles of the bursts exhibit a characteristic exponential decay, with frequency-dependent decay rates (to be explained later in the section). This favours a damping mechanism inherent to the ambient plasma and hence plasma emission hypothesis for the bursts. The decay mechanism may be owing to collisional damping, Landau damping or the deflection time for streaming electrons.

The time-profile analysis involves determining the rise and decay times of the individual noise storm bursts. The rising phase does not display exponential growth; a better description would be that of a Gaussian profile, which usually fits the general behaviour of the burst profiles, often down to flux levels beyond which the decay becomes exponential. Standard Gaussians, of the form shown in equation (2), were fitted to the time profile of the bursts, each at their central frequency of emission. When classified based on the degree of deviation from these Gaussian profiles, the study reveals that, $\approx 38$ per cent of events had a suitable (symmetric) Gaussian threading the data points, and $\approx 62$ per cent with steep cut-offs along the leading (decay phase) or the trailing (rise phase) edges of the distribution. The plots in Fig. 4 explain the method invoked for the time-profile studies of Type I bursts. While subplots (a) and (d) trace a Gaussian distribution, subplots (b) and (c) have steeper distributions for their data points on the ascending and descending sections, respectively. 

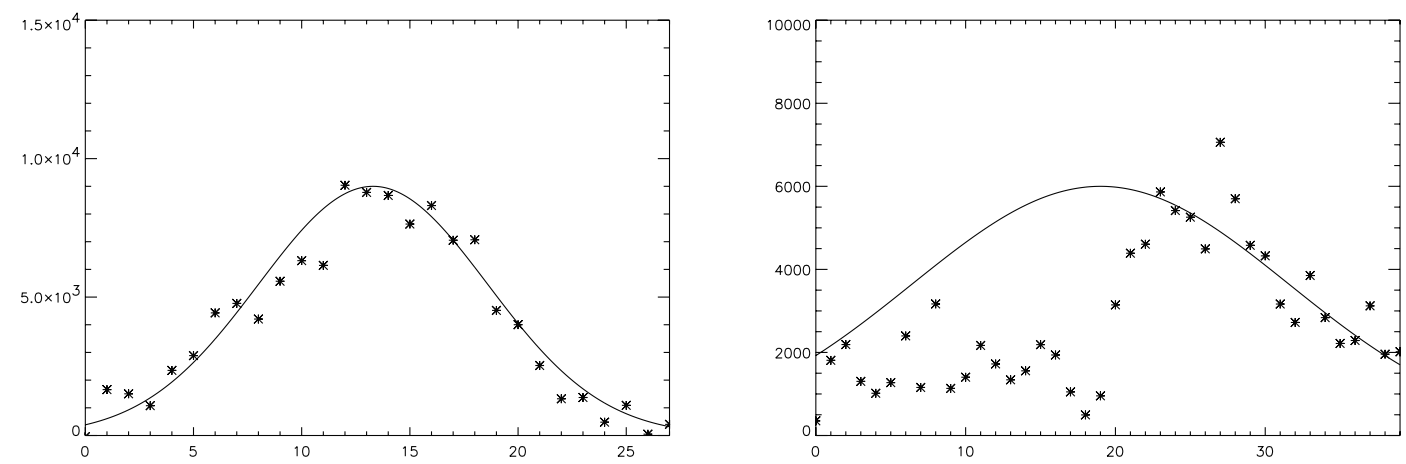

[a]

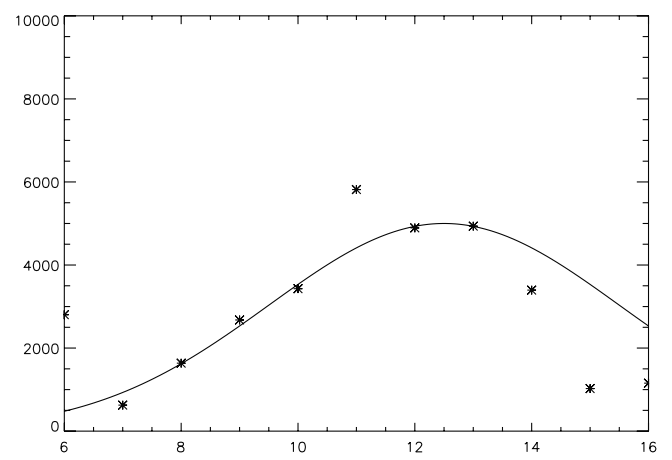

[b]

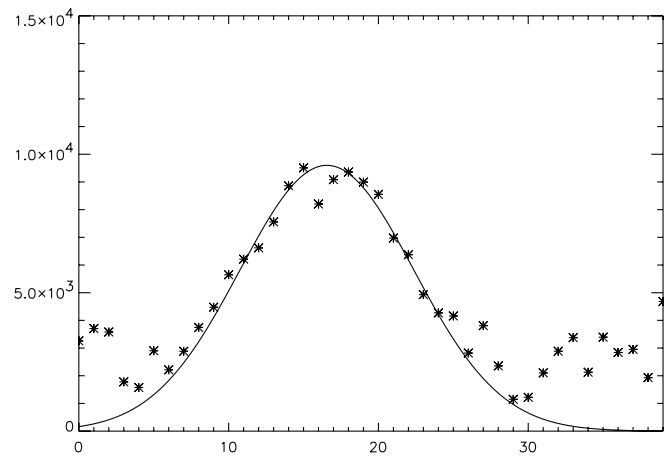

[c]

Figure 4. The three kinds of isolated Type I noise storm bursts, according to their averaged time profiles. The $x$ axis is in units of time, with the adjacent data points separated by $\approx 0.25 \mathrm{~s}$; the $y$ axis is in uncalibrated flux units. Subplots (a) and (d) indicate symmetry, while (b) and (c) show trailing and leading edge asymmetry, to the fitted Gaussian distribution.

The rise time $\left(t_{\mathrm{r}}\right)$ and the decay time $\left(t_{\mathrm{d}}\right)$ for each of the profiles, at their central frequency of emission, was determined based on the symmetric Gaussian profile fitted to each of the isolated noise storm burst events chosen. Using adequate parameters, the fittings give an excellent approximation to the real burst, ignoring the noise fluctuations. A smoothed minimum envelope was calculated and assigned as the mean local background to the observations. The background was then subtracted, taking care not to reduce the modulated flux of the individual bursts. Hence the times would now correspond to the extent of the half-power points in the actual data, from the symmetry axes of the fitted Gaussian profiles. $t_{\mathrm{r}}$ and $t_{\mathrm{d}}$ may be considered the characteristic time interval for the growth of plasma instabilities (Subramanian, Krishan \& Sastry 1981; Mészárosová et al. 2003 and references therein), and the damping of the plasma waves in the source region for the bursts, respectively. A plot of $\left(t_{\mathrm{r}}\right.$ versus $\left.t_{\mathrm{d}}\right)$, as shown in Fig. 5, has a wider dispersion of the points about the mean. The result shows that the majority of the burst events had either a longer phase of instability and became quickly damped, or vice versa. The scatter in the statistical value for the decay time, derived from all the bursts considered, may be owing to instabilities during the excitation or to peculiarities during the decay phase.

As a Gaussian curve extends to infinity on the decay portion of the time profile (tail of the distribution), a fitting using a true exponential-damping curve of the form

$\exp \left[\left(t-t_{0}\right) / \tau\right]$

where $\tau$ is the decay time-constant and $t_{0}$ is a fitting parameter, was attempted, which would offer a better description of the segment that occurs at the lower intensity level below the peak. A semilog plot of the intensity (or calibrated flux S) versus time of the e-fold decay

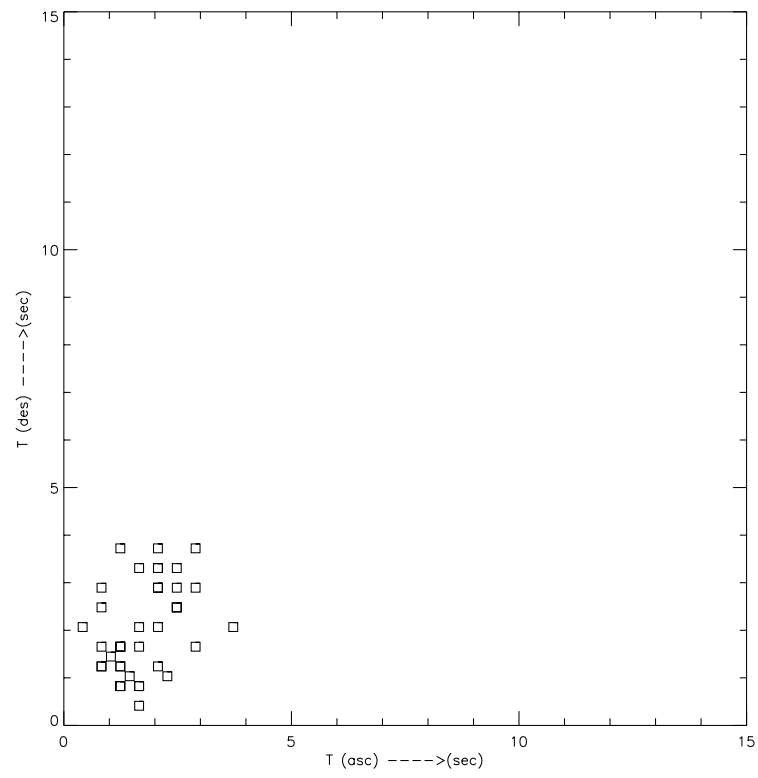

Figure 5. Scatterplot of ascending versus descending times of the isolated Type I noise storm bursts.

segment would then yield a linear result, whose slope determines the decay (or damping) constant $\tau\left(=-[\mathrm{d} \ln S / \mathrm{d} t]^{-1}\right)$.

The burst duration can be attributed to the lifetime of the coherent electron beam that excites the plasma oscillations (Takakura 1963). This lifetime is defined by the disruption in the orderly motion in the electron beam, caused by their collisions with, and deflection 


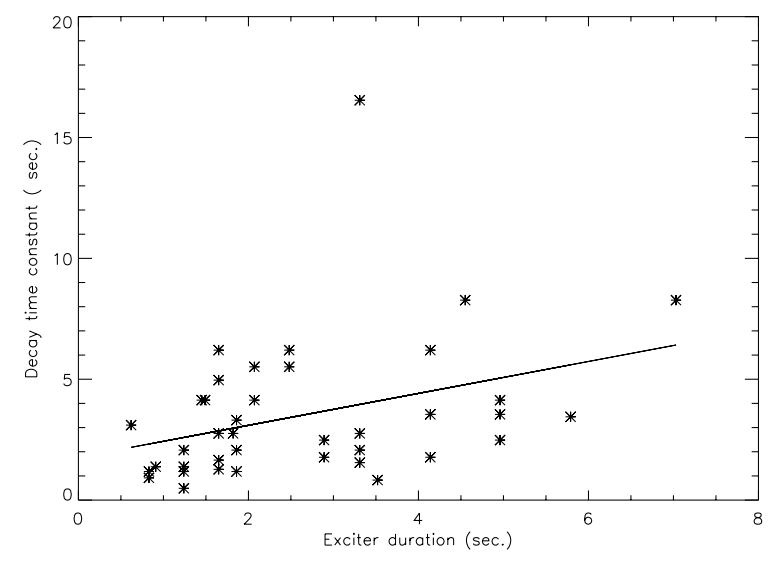

Figure 6. Scatterplot of exciter duration versus decay time constant for the isolated Type I noise storm bursts.

by, the thermal ions. The resultant energy loss by the electron beam excites coherent plasma-wave oscillations. Hence, the scale of the burst duration is comparable to this deflection time-scale, which in turn directly varies as the third power of the electron beam velocity, implying that the velocity of the exciting disturbance is quite small; conversely, the bursts themselves were generated in a region of complex, filamentary coronal magnetic field topology, where the thermal energies are relatively lower than that of the ambient coronal plasma. For bursts of shorter duration, the value of the electron beam velocity approaches the regime wherein the collisionless Landau damping becomes more pronounced.

In order to determine the actual damping phenomenon (collisional or Landau damping), an estimation of the electron-ion collision temperature $T_{\mathrm{c}}$ was performed, based on the expression (Aubier \& Boischot 1972; Güdel \& Benz 1990)

$T_{\mathrm{c}} \propto v^{4 / 3} \tau^{2 / 3} \mathrm{~K}$,

where $v$ is the central frequency $(\mathrm{MHz})$ of the isolated burst event and $\tau$ is the damping constant in seconds. Collisional damping is sensitive to the ambient plasma temperature which varies for different events. The values for $T_{\mathrm{c}}$ were found to vary from $1.5 \times 10^{6}$ to $1.3 \times 10^{7} \mathrm{~K}$ in the $30-130 \mathrm{MHz}$ range, evidently much less than the high brightness temperatures $\left(>10^{11} \mathrm{~K}\right)$ associated with noise storm bursts (Thejappa 1991). This, combined with the predominantly symmetrical burst distribution, as revealed from the frequency-profile studies (Fig. 5), and the failure of the collisional damping hypothesis to account for the decay rates of the bursts, lead to the reasoning that, the decay phase (tail of the exciter stream) is attributed to non-collisional damping mechanisms.

The Gaussian distribution offers an appropriate fitting to the time profiles of the bursts up to a point [that also serves to locate the extent of the exciter duration (Aubier \& Boischot 1972), in this case to an accuracy of $\pm 0.4 \mathrm{~s}$ ] beyond which the exponential decay law sets in. A scatterplot of the exciter duration versus the decay time constant, for the bursts considered here, is depicted in Fig. 6, along with a linear least-squares fitting. A positive correlation of $\sim 35$ per cent is found. This weak dependence of $\tau$ on the exciter duration, and the fact that an exponential decay law generally favours collisional damping of the plasma waves (Aubier \& Boischot 1972), along with a value of $\Delta r$ that falls short of those obtained from multifrequency imaging studies of Type I bursts (Thejappa 1991) (implying that collisions result in damping of the $\mathrm{L}$ waves at a much faster rate than the observed burst decay), could be indicative of the collisionless Landau damping phenomenon of $\mathrm{L}$ waves by the background plasma, especially at these metric wavelengths (Elgarøy 1961; Malville 1962; Zaitsev, Mityakov \& Rapoport 1972), not accounting for the case-sensitive nature of the very emission process of Type I bursts.

Applying the standard expressions for the group velocity $\left(v_{\mathrm{g}}\right)$ and Landau damping constant $\left(\gamma_{\mathrm{L}}\left(\equiv \tau^{-1}\right)\right.$ ), (Thejappa, Gopalswamy \& Kundu 1990; Thejappa 1991; Shanmugha Sundaram \& Subramanian 2004), and assuming values for the phase velocity $\left[v_{\mathrm{ph}}(\approx\right.$ $\left.\left.v_{T_{\mathrm{b}}}\right)\right]$ to be $10^{10} \mathrm{~cm} \mathrm{~s}^{-1}$ and $v_{\mathrm{T}} \approx 3.89 \times 10^{8} \mathrm{~cm} \mathrm{~s}^{-1}$, the estimated damping length $(\Delta r)$ is $\sim 1.1 \times 10^{-2} \mathrm{R} \odot \cdot v_{\mathrm{ph}} \approx v_{T_{\mathrm{b}}}$, when critical fluctuations in $T_{\text {eff }}$ of the $\mathrm{L}$ waves, at the threshold densities of the trapped electrons that lie proximal to the onset of plasma instabilities, rise steeply to the levels of $T_{\mathrm{b}}$ observed for Type I burst events.

\section{DISCUSSION AND CONCLUSION}

The existence of large, widely separated sunspot groups or active region (AR) complexes, with a high degree of complexity and strength in the associated magnetic topology, a large-scale reorganization of magnetic field structure in the photosphere and corona, of opposing polarity, interconnected by dense, expansive coronal magnetic arches, constitute the choicest of spatiotemporal correlations, for fundamental plasma frequency radiation associated with noise storms (Mercier 1984). The source region for the noise storms radiation is positioned along the magnetic loop that appears across bipolar ARs, and proximal to the apex of the loop, for the magnetic field in the leading spot of the region (Stewart 1985). Imaging and spectral observations of radio noise storms strongly suggest that there is a close relationship between bursts and energy release events at their source (Mercier \& Trottet 1997). From the observed fine spectrotemporal characteristics of isolated Type I bursts, it is likely that they are a signature of scattered small-scale sites of energy release giving rise to electron acceleration (Benz \& Wentzel 1981; Spicer, Benz \& Huba 1981). The sites are related to the locations of trapped electrons within the loop, and appear as bright regions in the soft $\mathrm{X}$-ray and extreme ultraviolet images, owing to the acceleration of these non-thermal electrons to energies of a few $\mathrm{keV}$ to few tens of $\mathrm{keV}$. This acts as a potential storehouse for persistent energy conversion and acceleration of the non-thermal electrons, contributing to the bursts of the noise storms at metric wavelengths. The discussions that follow have been vividly set to explain the varied causative mechanisms, any, or a few, or all of which could have a definite hand in the highly transient nature of noise storm bursts.

Type I noise storm bursts are structured in time, much akin to the decimetric spikes (Karlický, Sobotka \& Jiřička 1996; Bárta \& Karlický 2001); their morphological similarities having been well discussed in studies on the latter (see Zaitsev et al. 1972; Benz \& Thejappa 1988; Güdel \& Benz 1990; LaRosa, Moore \& Shore 1994; Krucker et al. 1995). One plausible reason for the fragmented appearance could be their largely stochastic origin. The effective temperature $\left(T_{\text {eff }}\right)$ of the $\mathrm{L}$ waves equates the observed brightness temperature $\left(T_{\mathrm{b}}\right)$ of the burst radiation, when the non-thermal electron beam density approaches a threshold density value. The values of $T_{\mathrm{b}}$, as estimated from the dynamic spectral data, are most likely produced by trapped electrons, when the ratio of number density of the superthermal electron beam to that of the ambient coronal plasma $\left(n_{b} / n_{e}\right)$ exceeds a critical threshold (Thejappa et al. 1990). In a trap, the presence of a loss cone would lead to the electron distribution turning anisotropic. At the threshold densities, $\left(T_{\text {eff }}\right)$ of the L waves rises steeply (Thejappa \& Kundu 1991; Thejappa 1991), 
and the anisotropic distribution of these energetic particles generate bursts at random. The effect is identical to the critical fluctuation in $T_{\text {eff }}$ of the $\mathrm{L}$ waves near the onset of a plasma instability. This threshold condition is governed by the stochastic nature of electron acceleration whereby, they are injected into the source region by collisionless shock waves, that move almost at Alfvén velocity (Thejappa 1987; Benz \& Thejappa 1988); hence the phenomenon of noise storm bursts exhibits sporadicity.

The nature of the fragmentation, of the kind observed as isolated type 1 bursts, could be owing to stochastic boundary or initial conditions of the respective processes, such as inhomogeneities in the coronal plasma, or a low-dimensional, non-linear deterministic process capable of generating such complicated patterns. Single bursts can be isolated, separated by quiet phases. The narrowband bursts show a tendency to be interrupted in their stationary phases, owing to short-term suppressions of the emission mechanism. The variability is caused by a stochastic input or a high-dimensional mechanism. In studies performed on the measured time-series of spikes, no low-dimensional behaviour was noticed (Isliker \& Benz 1994), implying their origin in complex processes with dimensions (degrees of freedom) from four to six, which includes infinity (stochasticity), because high-dimensional deterministic behaviour is hard to distinguish from stochasticity.

Suppression of the emission mechanism, for durations comparable to that observed in sporadic burst, could be explained as a consequence of either an inhomogeneous plasma flowing into a reconnection region, or activity at a shock front modulated by the upstream medium, creating cascading magnetohydrodynamic (MHD) turbulence in the plasma reconnection outflows (LaRosa et al. 1994; Sato, Matsumoto \& Nagai 1982). The influence of the MHD turbulence on the resulting radio emission is two-fold: (i) it chaotically changes the radio emission frequency; and (ii) the very fast plasma parameter changes in the radio source reduce or even stop the plasma instability under study, thereby effectively interrupting the radio emission (Karlický et al. 1996; Bárta \& Karlický 2001).

The emergence of new magnetic flux at sites of ARs causes an increase in the size of the coronal loops that connect regions of dissimilar polarity. The newly emerging magnetic flux, and its reconnection with the pre-existing flux lines, results in localized mass flow (McLean 1981); the ensuing weak, superAlfvénic shock causes wake microturbulence (Spicer et al. 1981; Melrose 1980a), leading to the excitation of plasma waves, within a few hours of their emergence at the ARs. The transformation of these high-frequency Langmuir (L) waves into transverse electromagnetic (TEM) waves occurs as a result of their scattering and coalescence, either on ion density fluctuations above the sites of loop reconnections and along the neutral-line current sheet or with the lower-hybrid waves excited by the trapped superthermal ions with a loss cone, that are borne out of weak collisionless shocks, generated owing to flux emergence (Wentzel 1985; Melrose 1980a). The reconnection loops need to be sufficiently dense and reach the level of the local plasma frequency, for the ambient coronal plasma to behave as a source region for the escape of Type I bursts.

Observations of bursts of Type I noise storms, at high frequency and time resolution, have conclusively shown their origin as a consequence of the non-thermal plasma emission mechanism. Analyses of the frequency and time profiles of bursts, which are a product of non-linear plasma instabilities in the source region and governed by laws of stochastics, reveal significant insight to the sites of largescale magnetic reorganization and reconnection in the outer solar corona. A drift-free nature for the narrowband emission is a sign of high-degree homogeneity in the source region of bursts, while the largely asymmetric time profile distribution reflects a tussle between growth and decay of plasma-wave instabilities in the turbulent outer corona.

The distribution of bursts with central-frequency, bandwidth, duration and peak radio flux, indicate that isolated Type I noise storm bursts are intense, short duration and narrow bandwidth events, generated at source regions that have a low degree of non-uniformity - regions that have a smoothly varying density, temperature and magnetic field structure. Studies on the narrow bandwidth of the isolated bursts conclude that, the dimension of the exciting disturbance is of lesser extent along the direction of the magnetic field lines, implying a smaller velocity dispersion and a near constant density and temperature structure. This reveals the high degree of uniformity in the source region for the noise storm bursts; and as the corona above the ARs is in a high state of inhomogeneity, the source region for bursts needs to be of a relatively smaller spatial extent.

Possible alternative explanations, for the weak dependence of $\tau$ on the exciter duration would be cases wherein, for the same exciter length, their velocities are larger, they encountered large density gradients along their trajectory (small duration for the exciter, or lower temperatures) - verifiable from white-light coronograph observations, or the turbulent bandwidth (exciter duration) is larger at increased coronal temperatures. From the physical emission process point of view we have the following.

(i) The exciter has a finite length as it travels through the corona. The time profile would then reflect the crossing of the exciter through the layer corresponding to the observed channel bandwidth (assuming a plasma hypothesis).

(ii) The exciting agent emits simultaneously on all frequencies where the burst is observable corresponding to a plasma layer of given height. The exciter function then would represent purely a temporal description of the emission process.

(iii) The time profile may be a combination of both: if a travelling disturbance starts a characteristic emission process going on even after the crossing of the agent through the observed layer, the resulting time profile could be a convolution of the time profile of the stimulation agent and the induced emission.

The total bandwidth of observed bursts is found to be 2-5 per cent of the central frequency; hence, the longitudinal extent of the emission region of a burst is several times less than the total beam length, assuming radiation to be near the local plasma frequency. This suggests that the beam emits only in a narrow region. After traversing it, the beam may either have decayed to a stable distribution, or the medium is unable to produce radio waves. AS the burst emission is triggered by a beam of superthermal electrons propagating through the corona (with a spread in their velocities), whose density exceeds a certain threshold leading to plasma instabilities at the source region, the reason for the cessation of bursts could be owing to stabilization in the causative electron beam as a result of non-uniformity in velocity (Landau damping of plasma waves owing to an excess of electrons with velocities that are smaller than the phase velocity of the wave) and density profile (Zaitsev et al. 1972), which in themselves are strongly governed by stochastic considerations.

The details on Type I emission process are expected to critically depend on (i) local physical conditions in the emitting sources, which are likely to vary with the ARs, and even within the magnetic field structure associated with one given AR; (ii) the spread in intensity of the bursts' emission itself. Thus, if the distribution of their peak flux densities is strongly affected by the emission process, 
it would change significantly with the storm event and frequency. Such observable characteristics lead to a fair speculation as to the peak flux density of each burst being nearly linearly related to the energy transferred to the non-thermal electrons. Characteristics like stationarity, intermittency and dimensionality need to be taken into account, in order to interpret the free magnetic energy release mechanism in the solar corona owing to the noise storm bursts. Added information regarding the plasma structure in the emission region and the spectral behaviour of the bursts would be an essential pre-requisite for such a study.

\section{ACKNOWLEDGMENTS}

We wish to thank E. Ebenezer of the Indian Institute of Astrophysics for system implementation and data archival in the context of the HRS. We also thank the referee for the effort and insightful comments that have improved the clarity in content and presentation of this paper. We are immensely grateful to the scientific and technical staff of the Gauribidanur Radio Observatory, jointly run by the Raman Research Institute and the Indian Institute of Astrophysics, for duly maintaining the scientific facilities on site.

\section{REFERENCES}

Alexander J. K., Brown L. W., Clark T. A., Stone R. G., Weber R., 1969, ApJ, 157, L163

Aubier M., Boischot A., 1972, A\&A, 19, 343

Bárta M., Karlický M., 2001, A\&A, 379, 1045

Benz A. O., Thejappa G., 1988, A\&A, 202, 267

Benz A. O., Wentzel D. G., 1981, A\&A, 84, 100

Brown L. W., 1973, ApJ, 180, 359

Cane H. V., 1979, MNRAS, 189, 465

Dulk G. A., Erickson W.C., Manning R., Bougeret J.-L., 2001, A\&A, 365, 294

Elgarøy Ø., 1961, Astrophys. Norv., 7, 123

Elgarøy Ø., 1977, Solar Noise Storms. Pergamon Press, London

Elgarøy Ø., Eckhoff H. K., 1966, Astrophys. Norv., 10, 127

Güdel M., Benz A. O., 1990, A\&A, 231, 202

Hey R., 1946, Nat, 157, 47

Isliker H., Benz A. O., 1994, A\&A, 285, 663

Kai K., Melrose D. B., Suzuki S., 1985, Solar Radiophysics: Studies of Emission from the Sun at Metre Wavelengths. A87-1385103-9. Cambridge University Press, Cambridge, pp. 415-441

Karlický M., Sobotka M., Jiřička K., 1996, Solar Phys., 168, 375

Kerdraon A., 1973, A\&A, 27, 361

Krucker S., Benz A. O., Aschwanden M. J., Bastian T. S., 1995, Solar. Phys., 160,151

Krüger A., 1979, Introduction to Solar Radio Astronomy and Radio Physics. Reidel Publishing Company, Dordrecht, p. 126

Kundu M. R., 1965, Solar Radio Astronomy. Wiley, New York, p. 443

LaRosa T. N., Moore R. L., Shore S. N., 1994, ApJ, 425, 856

McLean D. J., 1981, Publ. Astron. Soc. Australia, 4, 132

Malville J. M., 1962, ApJ, 136, 266

Mangeney A., Veltri P., 1976, A\&A, 47, 181

Melrose D. B., 1980a, Solar Phys., 67, 357

Melrose D. B., 1980b, Space Sci. Rev., 26, 3

Melrose D. B., Stenhouse J. E., 1977, Australian J. Phys., 30, 481

Mercier C., Trottet G., 1997, ApJ, 474, L65

Mészárosová H. et al., 2003, A\&A, 407, 1115

Novaco J. C., Brown L. W., 1978, ApJ, 221, 114

Ramesh R., Subramanian K. R., Sundarajan M. S., Sastry Ch. V., 1998, Solar Phys., 181, 439

Sastry Ch. V., 1969, Solar Phys., 10, 429

Sato T., Matsumoto H., Nagai K., 1982, JGR, 87, 6089

Shanmugha Sundaram G. A., Subramanian K. R., 2004, ApJ, 605, 948
Spicer D. S., Benz A. O., Huba J. D., 1981, A\&A, 105, 221

Stewart R. T., 1985, Solar Phys., 96, 381

Subramanian K. R., Krishan V., Sastry Ch. V., 1981, Solar Phys., 70, 375

Takakura T., 1963, Publ. Astron. Soc. Japan, 15, 327

Taylor J. D., ed., 1995, Introduction to Ultra-Wideband Radar Systems. CRC Press, Boca Raton, Florida, p. 3

Thejappa G., 1987, Solar Phys., 111, 45

Thejappa G., 1991, Solar Phys., 132, 173

Thejappa G., Kundu M. R., 1991, Solar Phys., 132, 155

Thejappa G., Gopalswamy N., Kundu M. R., 1990, Solar Phys., 127, 165

Warwick J. W., Dulk G. A., 1969, ApJ, 158, L123

Wentzel D. G., 1985, ApJ, 296, 278

Wild J. P., 1950, Australian J. Sci. Res., A4, 36

Wild J. P., McCready L. L., 1950, Australian J. Sci. Res., A3, 387

Yates K. W., Wielebinski R., 1966, Australian J. Phys., 19, 389

Zaitsev V. V., Mityakov N. A., Rapoport V. O., 1972, Solar Phys., 24, 444

\section{APPENDIX A: CALIBRATION OF THE DYNAMIC SPECTRUM USING THE GALACTIC BACKGROUND}

The method employed in the calibration of the flux densities of Type I bursts, over the entire frequency range of the spectrograph, to an absolute scale, is relevant to low-frequency ( $\leqslant 100 \mathrm{MHz})$ radio telescopes, with inherent low angular resolution, used in spectral observations of solar radio bursts, with the galactic background radiation as the principal contributor (in comparison to receiver noise) to the system temperature. The antenna response to the brightness distribution of the galactic background radiation is a function of its angular resolution and observing frequency. The spectrum of this unpolarized galactic background has been well catalogued (Yates \& Wielebinski 1966; Alexander et al. 1969; Brown 1973; Novaco \& Brown 1978; Cane 1979), in either of the galactic hemispherical regions, and serves as a highly reliable calibrator source at metric and decametric (1-100 MHz) wavelengths. As radio signals from the Sun and the galactic plane essentially arrive along the same path, in their approach to the receiving system, this technique ensures that the errors and losses introduced along the signal path (like impedance or ohmic losses in the antenna system) are effectively identical in either case. This scheme is also adept at correcting for ionospheric absorption and partial ground reflection of the celestial radio signals (Dulk et al. 2001), to an accuracy of 2 per cent, and is especially suited for spatially low-resolving telescopes, that have difficulty in discerning the fine spatial structures in standard, unresolvable radio sources, for use in absolute calibration of radio data.

For antennas with low directivity, the galactic background radiation is essentially isotropic. The solid angle of the group beam of the array is $0.56-0.13 \mathrm{sr}$ in the $30-130 \mathrm{MHz}$ range, while it is $1.64 \mathrm{sr}$ for the individual LPDAs in the group. The large value for beam width implies that, variability in the galactic background with local sidereal time (LST), as contributed by the less intense, isotropic, large-scale features, remain minimal, with the variations in sky temperature being $<30$ per cent (Yates \& Wielebinski 1966) at high galactic latitudes.

The spectra of the galactic background radiation, at the local meridian-transit point, were obtained from observing regions that were far removed from the galactic plane - ideally towards the North Galactic Pole (NGP) and the South Galactic Pole (SGP); thereby large changes in the brightness distributions owing to structures [like partly ionized hydrogen (HI) in the interstellar medium] located in the galactic plane, get excluded. The NGP(J2003.0: $\left.\alpha=12^{\mathrm{h}} 51^{\mathrm{m}} 33^{\mathrm{s}} .9675, \delta=+27^{\circ} 06^{\prime} 49^{\prime} .3824\right)$ was observed on 2002 
December 25 and the SGP(J2003.0: $\alpha=00^{\mathrm{h}} 51^{\mathrm{m}} 33^{\mathrm{s}} .9675, \delta=$ $\left.-27^{\circ} 06^{\prime} 49^{\prime} .3824\right)$ the previous day, the former at early dawn $(\mathrm{LST}=11: 21: 34 ; \mathrm{IST}=05: 28: 30)$ and the latter around local sunset $(\mathrm{LST}=23: 21: 34$; IST $=17: 30: 27)$, in the transit mode. This observation schedule augured well with the location coordinates and beam width of the LPDAs in the four-antennas group, as to pursue the observations and determine the spectrum in the direction of the two poles. The observing epoch was carefully chosen to ensure that the Sun had a minimal influence in the primary beam response. Considering the tapering-off of gain (owing to the array-pattern response) with angular distance away from the local meridian at zenith, a symmetrical observing window of about $3 \mathrm{~h}$ was chosen. As the presence of large sidelobes, especially at the lower frequencies of the observing spectral range, would lead to the acquisition of remnant solar flux proximal to the horizon, only $1 \mathrm{~h}$ of the galactic background about the transit point remained utilizable in the calibration of solar data. The wider beam along the E-plane meant that 'boresight' alignment to declination values for the poles alone needed to be set, so that the antenna temperature is contributed predominantly by the brightness distribution of the galactic background. Results from observations of the galactic background during the course of the day, at either of the poles, were found to have good agreement with similar data published from observations done previously.

The availability of $1 \mathrm{~h}$ of data on the calibration source, symmetrical about the transit point, set an identical limit on utilization of the solar spectral data, for the specific days of observation. The gain across all the 401 frequency channels was found to have reasonable stability. With a knowledge of the deviation of the observed spectrum from the catalogued spectra of the galactic background at identical LSTs, and the antenna gain in the direction of the radio source, the antenna temperature was converted to the noise storm flux density values. For the total power receiver case, the detected signal is a convolved response of the antenna power pattern and the sky brightness distribution, across a channel width of $250 \mathrm{kHz}$, termed the spectral power, measured in units of $\mathrm{mV}^{2} \mathrm{~Hz}^{-1}$. The spectral power is then transformed to units of radio-flux density (measured in $\mathrm{W} \mathrm{m}^{-2} \mathrm{~Hz}^{-1}$ ), given the data on the spectral power of noise storms and the galactic background, and the model for radio-flux density of the latter.

This paper has been typeset from a $\mathrm{T}_{\mathrm{E}} \mathrm{X} / \mathrm{L} \mathrm{A} \mathrm{T}_{\mathrm{E}} \mathrm{X}$ file prepared by the author. 